\title{
Sodium-glucose co-transporter-2 inhibitors: know the patient and the drugs
}

\author{
LL Lim ${ }^{1,2,3}$, MB, BS, MRCP, Juliana CN Chan ${ }^{1,3,4,5}$ *, MD, FRCP \\ ${ }^{1}$ Department of Medicine and Therapeutics, The Chinese University of Hong Kong, Prince of Wales Hospital, Hong Kong \\ ${ }^{2}$ Department of Medicine, Faculty of Medicine, University of Malaya, Kuala Lumpur, Malaysia \\ ${ }^{3}$ Asia Diabetes Foundation, Hong Kong \\ ${ }^{4}$ Hong Kong Institute of Diabetes and Obesity, The Chinese University of Hong Kong, Prince of Wales Hospital, Hong Kong \\ ${ }^{5} \mathrm{Li}$ Ka Shing Institute of Health Sciences, The Chinese University of Hong Kong, Prince of Wales Hospital, Hong Kong \\ *Corresponding author: jchan@cuhk.edu.hk
}

Hong Kong Med J 2019;25:268-70

https://doi.org/10.12809/hkmj195089

Timely intensification of glucose-lowering drugs in type 2 diabetes mellitus (T2DM) is essential to improve durability of glycaemic control and prevent diabetes-related complications. ${ }^{1,2}$ Progressive betacell failure is a hallmark in T2DM, especially in Asians in whom pancreatic beta-cell dysfunction and insulin resistance frequently coexist. ${ }^{3,4}$ In the Hong Kong Diabetes Register, 50\% of patients with T2DM were treated with insulin after 10 years of disease. ${ }^{5}$ Despite a growing portfolio of glucoselowering drugs in the last decade, ${ }^{6}$ only one third of patients with type 1 diabetes mellitus (T1DM) or T2DM achieved personalised glycaemic goals.? Although increasing insulin dosages may improve glycaemic control, overzealous use of insulin can increase the risk of hypoglycaemia and weight gain.? Weight gain leads not only to higher insulin dosages but also to increased blood pressure, which is a major cardiovascular risk factor and attenuates the benefits of glucose lowering. ${ }^{8}$

Sodium-glucose co-transporter-2 (SGLT2) inhibitors reduce blood glucose by inhibiting glucose reabsorption in the early proximal renal tubule and promote glucosuria. While the calorie loss can lead to weight reduction, the coupling of sodium and glucose transporters also leads to natriuresis which contribute to lowering blood pressure. ${ }^{3,9,10}$ Given its beneficial effects on multiple cardiovascular risk factors, there is a strong rationale for using this class of medications as an insulin-sparing agent. ${ }^{2,11}$

In this issue of Hong Kong Medical Journal, Tan et $\mathrm{al}^{12}$ provide practical guidance to help physicians recognise, monitor, and treat patients with SGLT2 inhibitors, in combination with insulin therapy. Compared with placebo, the addition of SGLT2 inhibitor to insulin therapy in patients with T1DM and $\mathrm{T} 2 \mathrm{DM}$ reduced haemoglobin $\mathrm{A} 1 \mathrm{c}$ by $0.4 \%$ to $0.7 \%$, body weight by 0.2 to $3 \mathrm{~kg}$, and total daily insulin dose by 0.2 to 13 units. ${ }^{12}$ Possible reasons for the low reported risk of hypoglycaemia with this combination therapy include: a compensatory increase in SGLT1mediated glucose reabsorption in the distal part of proximal renal tubule; the upregulation of counterregulatory mechanisms including increase in glucagon and hepatic gluconeogenesis; and reduced glycaemic variability. ${ }^{9,12,13}$

In patients with T2DM with cardiovascularrenal complications and/or multiple risk factors, data from randomised controlled trials have confirmed the benefits of SGLT2 inhibitors in reducing major adverse cardiovascular events, hospitalisation for heart failure, all-cause death, and worsening renal function including end-stage renal disease over a median follow-up period of between 2.6 and 4 years. ${ }^{14-17}$ In addition to lowering blood glucose, blood pressure, and body weight, SGLT2 inhibitors may also increase blood haemoglobin with increased tissue oxygenation and decrease uric acid, a known cardiovascular risk factor. ${ }^{18}$

Another mechanism that may explain the cardiovascular-renal benefits of SGLT2 inhibitors is a metabolic switch, in part due to increase in glucagon, from glucose to free fatty acid oxidation with increased formation of ketone bodies as a more efficient energy source. ${ }^{10}$ In non-stressed situation, use of SGLT2 inhibitors can be associated with physiological ketosis but without acidosis. However, in the presence of metabolic stress such as surgical procedures and critical illnesses, especially in patients who are lean and those with reduced betacell reserves due to long disease duration as well as those who take ketogenic diet for weight reduction, overt/euglycaemic DKA may occur.

In order to minimise the risk of hypoglycaemia, Tan et $a^{12}$ suggest down-titration of total daily insulin dose by $10 \%$ to $20 \%$. Depending on the general state of the patients, treatment modifications should be individualised with reinforcement of sickday management including increased frequency of monitoring of blood glucose and blood/urine ketone. ${ }^{11}$ Adequate communication between patients and physicians is particularly important during the perioperative or periprocedural periods where close adherence to treatment recommendations including temporary withdrawal of SGLT2 inhibitors is necessary. During these periods of major stress, increased release of counterregulatory hormones coupled with reduced beta-cell release, against 
a background of increased glucagon release, can markedly increase the risk of overt/euglycaemic DKA in patients treated with SGLT2 inhibitors. Ensuring adequate hydration, avoiding low carbohydrate diet, and ensuring adequate coverage of insulin are needed to avoid metabolic decompensation. ${ }^{11,12}$

Despite the high relative risk, the absolute incidence of urogenital infections associated with the use of SGLT2 inhibitors is low and usually well tolerated and self-limiting, at least in randomised controlled trial settings. ${ }^{12}$ However, the potential link between the use of SGLT2 inhibitors and Fournier gangrene, a progressive bacterial necrotising fasciitis of the perianal, perineal, and/or external genital areas is concerning. ${ }^{19}$ Despite its rare occurrence affecting less than $0.02 \%$ of hospitalisations in the US, these events are extremely devastating and distressing to patients and can be potentially fatal. ${ }^{19}$ In real-world settings where care is less well supervised, poor glycaemic control may persist even with the use of SGLT2 inhibitors, especially in patients with poor insulin reserve but not adequately replaced. Indeed, in patients who developed Fournier gangrene, as many as $70 \%$ had poor glycaemic control and/or obesity. ${ }^{19}$ In these patients, the glucosuric milieu induced by SGLT2 inhibitors in these anatomical sites with rich bacterial flora may increase the risk of Fournier gangrene. ${ }^{19,20}$

Based on data from the US Food and Drug Administration Adverse Event Reporting System (FAERS), 55 patients who were treated with SGLT2 inhibitors developed Fournier gangrene during a 6-year period, compared with 19 patients treated with other glucose-lowering drugs. ${ }^{20}$ Physicians must emphasise the importance of good personal hygiene when using SGLT2 inhibitors, especially in those with poor glycaemic control. ${ }^{3,11}$ A high index of suspicion for the condition is required if patients complain of local pain disproportionate to findings on physical examination, especially in those with risk factors such as long-term glucocorticoid therapy, immunocompromised state, and chronic alcoholism. ${ }^{19,20}$ If diagnosed early, Fournier gangrene is treatable with withdrawal of SGLT2 inhibitors, fluid resuscitation, immediate broad-spectrum antibiotics, and urgent surgical debridement. ${ }^{19}$

Another safety concern associated with the use of SGLTs is lower extremity amputation (LEA). ${ }^{15,17,21}$ Using pharmacovigilance data from the US FAERS, canagliflozin, with or without concomitant insulin therapy, was associated with excess risk of LEA; no similar association was recorded for dapagliflozin or empagliflozin..$^{22}$ In the Swedish and Norwegian national health registers, the relative risk of LEA increased by 2 times with the use of SGLT2 inhibitors compared with glucagon-like peptide 1 receptor agonists, irrespective of history of cardiovascular disease or amputation, although the overall event rate was low ( 2.7 vs 1.1 events per 1000 person-years). ${ }^{23}$ More studies are needed to clarify whether the risk of LEA is a class effect or drug-specific, as well as to reveal the underlying mechanisms, clinical profiles of patients, and settings of these clinical events. Examination of lower extremity including foot pulses is particularly important, especially in those with multiple risk factors, history of foot ulcers, and/or dehydrated (eg, high-dose diuretics) in whom SGLT2 inhibitors should be used with caution or avoided altogether.

In day-to-day practice, the key questions for patients and physicians are when and how to safely initiate SGLT2 inhibitors as adjunctive to insulin therapy. The clinical perspectives by Tan et $\mathrm{al}^{12}$ contextualises the patient profiles and provides practical tips to avoid adverse events. The large body of evidence supports the importance of periodic assessment of risk factors and complications and use of personalised data to stratify risk, educate/ empower patients, and promote good patient-doctor communication to maximise benefits and minimise harms of SGLT2 inhibitors in the prevention of morbidities, hospitalisations, and premature death related to $\mathrm{T} 2 \mathrm{DM} .^{24}$

\section{Author contributions}

All authors contributed to the concept or design, data interpretation, drafting of the article, and critical revision for important intellectual content. All authors contributed to the manuscript, approved the final version for publication, and take responsibility for its accuracy and integrity.

\section{Funding/support}

This work received no specific grant from any funding agency in the public, commercial, or not-for-profit sectors.

\section{Conflicts of interest}

JCN Chan is the Chief Executive Officer (on pro-bono basis) of Asia Diabetes Foundation, a charitable foundation established under The Chinese University of Hong Kong Foundation for developing the JADE Technology. She has received honoraria and travelling support for consultancy or giving lectures and her affiliated institutions have received research and educational grants from Amgen, Ascencia, AstraZeneca, Bayer, Bristol-Myers Squibb, Boehringer Ingelheim, DaiichiSankyo, Eli-Lilly, GlaxoSmithKline, Medtronic, Merck Serono, Merck Sharp \& Dohme, Novo Nordisk, Pfizer, and Sanofi. LL Lim has received honoraria and travelling support for giving lectures and her affiliated institutions have received research and educational grants from AstraZeneca, Boehringer Ingelheim, Merck Serono, Merck Sharp \& Dohme, Novartis, Novo Nordisk, Pfizer, Procter \& Gamble, Sanofi, and Servier.

\section{References}

1. Ray KK, Seshasai SR, Wijesuriya S, et al. Effect of intensive control of glucose on cardiovascular outcomes and death in patients with diabetes mellitus: a meta-analysis of randomised controlled trials. Lancet 2009;373:1765-72. 
2. Davies MJ, D’Alessio DA, Fradkin J, et al. Management of hyperglycemia in type 2 diabetes, 2018. A consensus report by the American Diabetes Association (ADA) and the European Association for the Study of Diabetes (EASD). Diabetes Care 2018;41:2669-701.

3. Lim LL, Tan AT, Moses K, Rajadhyaksha V, Chan SP. Place of sodium-glucose cotransporter-2 inhibitors in East Asian subjects with type 2 diabetes mellitus: Insights into the management of Asian phenotype. J Diabetes Complications 2017;31:494-503.

4. Yabe D, Seino Y. Type 2 diabetes via $\beta$-cell dysfunction in east Asian people. Lancet Diabetes Endocrinol 2016;4:2-3.

5. Tong PC, Ko GT, So WY, et al. Use of anti-diabetic drugs and glycaemic control in type 2 diabetes-The Hong Kong Diabetes Registry. Diabetes Res Clin Pract 2008;82:346-52.

6. Chatterjee S, Khunti K, Davies MJ. Type 2 diabetes. Lance 2017;389:2239-51

7. Aschner P, Gagliardino JJ, Ilkova HM, et al. Nonachievement of glycemic target-results from the International Diabetes Management Practices Study (IDMPS). Diabetes 2018;67(Supplement 1):1030-P.

8. Genev NM, Lau IT, Willey KA, et al. Does insulin therapy have a hypertensive effect in type 2 diabetes? J Cardiovasc Pharmacol 1998;32:39-41.

9. Rieg T, Vallon V. Development of SGLT1 and SGLT2 inhibitors. Diabetologia 2018;61:2079-86.

10. Lytvyn Y, Bjornstad P, Udell JA, Lovshin JA, Cherney DZ Sodium glucose cotransporter-2 inhibition in heart failure: Potential mechanisms, clinical applications, and summary of clinical trials. Circulation 2017;136:1643-58.

11. Deerochanawong C, Pheng CS, Matawaran BJ, et al. Use of SGLT-2 inhibitors in patients with type 2 diabetes mellitus and multiple cardiovascular risk factors: an Asian perspective and expert recommendations. Diabetes Obes Metab 2019 Jul 2. Epub ahead of print.

12. Tan K, Chow WS, Leung J, et al. Clinical considerations when adding a sodium-glucose co-transprter-2 inhibitor to insulin therapy in patients with diabetes mellitus. Hong Kong Med J 2019;25:312-9.

13. Rama Chandran S, Tay WL, Lye WK, et al. Beyond HbA1c: Comparing glycemic variability and glycemic indices in predicting hypoglycemia in type 1 and type 2 diabetes. Diabetes Technol Ther 2018;20:353-62.

14. Zinman B, Wanner C, Lachin JM, et al. Empagliflozin, cardiovascular outcomes, and mortality in type 2 diabetes. $\mathrm{N}$ Engl J Med 2015;373:2117-28.

15. Neal B, Perkovic V, Mahaffey KW, et al. Canagliflozin and cardiovascular and renal events in type 2 diabetes. $\mathrm{N}$ Engl J Med 2017;377:644-57.

16. Wiviott SD, Raz I, Bonaca MP, et al. Dapagliflozin and cardiovascular outcomes in type 2 diabetes. N Engl J Med 2019;380:347-57.

17. Perkovic V, Jardine MJ, Neal B, et al. Canagliflozin and renal outcomes in type 2 diabetes and nephropathy. N Engl J Med 2019;380:2295-306.

18. Inzucchi SE, Zinman B, Fitchett D, et al. How does empagliflozin reduce cardiovascular mortality? Insights from a mediation analysis of the EMPA-REG OUTCOME trial. Diabetes Care 2018;41:356-63.

19. Hagedorn JC, Wessells H. A contemporary update on Fournier's gangrene. Nat Rev Urol 2017;14:205-14

20. Bersoff-Matcha SJ, Chamberlain C, Cao C, Kortepeter C, Chong WH. Fournier gangrene associated with sodiumglucose cotransporter-2 inhibitors: A review of spontaneous postmarketing cases. Ann Intern Med 2019 May 7. Epub ahead of print.

21. Ryan PB, Buse JB, Schuemie MJ, et al. Comparative effectiveness of canagliflozin, SGLT2 inhibitors and nonSGLT2 inhibitors on the risk of hospitalization for heart failure and amputation in patients with type 2 diabetes mellitus: A real-world meta-analysis of 4 observational databases (OBSERVE-4D). Diabetes Obes Metab 2018;20:2585-97.

22. Fadini GP, Avogaro A. SGLT2 inhibitors and amputations in the US FDA Adverse Event Reporting System. Lancet Diabetes Endocrinol 2017;5:680-1.

23. Ueda P, Svanström H, Melbye M, et al. Sodium glucose cotransporter 2 inhibitors and risk of serious adverse events: nationwide register based cohort study. BMJ 2018;363:k4365.

24. Lim LL, Lau ES, Kong AP, et al. Aspects of multicomponent integrated care promote sustained improvement in surrogate clinical outcomes: A systematic review and meta-analysis. Diabetes Care 2018;41:1312-20. 